\title{
Electrical discharge machining of MMCs reinforced with very small particles
}

\author{
A. Pramanik \\ Department of Mechanical Engineering, Curtin University, Western Australia 6102 \\ Phone: +61 89266 7981, Fax: +61 89266 2681, Email: alokesh.pramanik@curtin.edu.au
}

\begin{abstract}
This paper investigates material removal rate (MRR), kerf width, surface finish and electrode wire wear for different pule-on-times as well as wire tensions during EDM of 6061 aluminium alloy reinforced with 10 vol\% $700 \mathrm{~nm}$ SiCp MMC. Effects of pulse-on-time on output variables at lower and higher wire tensions were investigated. Similarly, effects of the wire tensions on output variables at shorter and longer pulse-on-times were also investigated. Longer pule-on-time increases the MRR though the higher wire tension reduces the MMR. The effect of wire tension on MRR is much more significant at longer pule-on-time compare to that at shorter pule-on-time. There is an optimum pule-on-time for which best surface finish is achieved. The surface finish deteriorates when the pulse-on-time is higher or lower than the optimum pule-on-time. With the rise of tension in wire, the surface roughness increases and decreases at shorter and longer pule-on-times respectively. The machined surface contains solidified molten material, splash of materials and blisters. Generation of the tapered slot with higher kerf width at the top indicates the wear of wire electrode. Significant variation of the electrode wire diameter was due to coating of the matrix, wear and clogging of small reinforced particles in the electrode gap.
\end{abstract}

Keywords: EDM, composites, aluminium, alloys, ceramics, roughness, speed, wear, particles

\section{Introduction}

Machining processes remove unwanted material from a bulk workpiece and introduce the shape of the final product [1]. In conventional machining, the material is reshaped by sharp cutting tools [2]. The complex deformation mechanism [3,4] of metal matrix composites (MMCs) causes high tool wear during traditional machining. Electrical discharge machining (EDM) is an extensively used non-traditional material shaping technique which is capable to process materials by high thermal energy due to electric discharge [5-7]. EDM process takes place in a dielectric fluid where the tool is one electrode has the shape of the cavity to be 
produced and the workpiece to be machined is the other electrode [8-10]. Electrically conductive materials irrespective of hardness are qualified to be machined using this method $[11,12]$. Thus, particle reinforced metal matrix material which is hard to machine by traditional methods has the potential to be machined by EDM [13-16]. MMCs are different from monolithic materials because of the reinforced ceramic particles. Therefore, it is very complicated to optimize the machining of MMCs and manufacture parts with low tolerance [17-19].

EDM process is capable to machine MMCs though the lower electrical conductivity of the reinforced particles affect the material removal rate. EDM produces microscopic craters on the machined surface because of random spark release [13, 20, 21]. Hung et al. [22] noted that only the current controls the surface roughness. The re-cast layer thickness rises with the rise of pulse-on-times and discharge current [20]. Gatto and Iuliano [23] performed roughing and finishing EDM on $2009 \mathrm{Al}$ alloy MMC where the reinforcements were $15 \% \mathrm{SiC}$ whiskers and $20 \% \mathrm{SiC}$ particles. It was noted that the surface roughness of $15 \%$ whiskers composite is worse than that of $20 \%$ particles composite. Rozenek et al. [24] studied the influence of pulse-on-time, voltage, pulse-off-time and current on the speed and roughness after WEDM of $\mathrm{Al}$ alloy reinforced with $\mathrm{Al}_{2} \mathrm{O}_{3}$ and $\mathrm{SiC}$ particles. The surface roughness and speed rise with the rise of voltage, released energy and pulse-on-time. Yan et al. [25] performed WEDM on 6061Al alloy reinforced with 10 and 20 vol.\% $\mathrm{Al}_{2} \mathrm{O}_{3}$ particle as well as 6061Al alloy. The machining speed of non-reinforced alloy was highest though similar machining speed was noted for $10 \%$ and $20 \%$ particle reinforced composites. The increase of the reinforcement volume fraction causes breakage of wire, narrower slit, rougher machined surface finish, and wider and deeper craters on the wire surface. The influence of volume percentages of reinforcement on EDM was also investigated by [26] and [21] who also noted the increase of MRR due to the rise of SiC reinforcement volume percentage. Seo, Kim et al. [21] also noted higher wear when volume percentage is lower which is contradictory to the results obtain by other researcher [26]. Saha et al. [27] tested EDM of in situ MMC where Fe was reinforced with 5 vol.\% TiC. They noted the TiC particles and a by-product $\mathrm{Fe}_{2} \mathrm{O}_{3}$ during EDM caused unstable machining. The effect of electrolyte concentration, current, pulse duration and voltage on MRR was studied by Liu et al. [13] during wire electrochemical discharge machining of 6061 aluminium alloy reinforced with $\mathrm{Al}_{2} \mathrm{O}_{3}$ particles MMC. Investigations similar to the above were also performed by Patel and Pandey et al.,[26] Agrawal et a.,[28], Guo et al. [29], Patil and Brahmankar [30], Manna and Bhattacharyya 
[31], Sivaprakasm et al. [32], Kumar et al., [33] Yue et al., [34], etc. where the range of parameters and MMCs were different.

The above discussion indicates that the progress on EDM of particle reinforced MMCs are very good and many interesting outcomes have been obtained in this field so far. However, the EDM of very small particle $(700 \mathrm{~nm})$ reinforced MMCs has not been considered yet though the smaller size of the reinforcement incorporates better mechanical properties in MMCs compare to that of bigger size of the reinforcement [35, 36]. There is a trend for MMCs to have smaller size of reinforced particles to widen its application. This suggests the importance to investigate the effect of EDM on MMCs with smaller reinforced particles. In addition the interacting effects of different input parameters have not been considered in any study yet. To address this issue, this study investigates EDM of MMCs reinforced with 0.7 $\mu \mathrm{m}$ particles. The material removal rates, surface finish, wear of the wire electrode and kerf/slit surface at different machining conditions have been investigated in details when input parameters and interacting at lower and upper levels.

\section{Materials and methods}

A series of wire electrical discharge machining (WEDM) was performed on FANUC ROBOCUT $\alpha$-0iD machine. The workpiece material was metal matrix composite of $10 \mathrm{vol} \%$ $\mathrm{SiC}$ particles of $700 \mathrm{~nm}$ size (approximately) reinforced 6061 aluminium alloy. The machining conditions are given in table 1 . The fixed parameters during machining were: wire speed $10 \mathrm{~m} / \mathrm{min}$, flushing rate $10 \mathrm{l} / \mathrm{min}$, open circuit voltage $85 \mathrm{~V}$, servo voltage $44 \mathrm{~V}$ and zinc coated $0.25 \mathrm{~mm}$ diameter brass wire. A $10 \mathrm{~mm}$ long slot was produced on a plate of $137 \times 42 \times 9 \mathrm{~mm}^{3}$ in each experiment. Two important parameters pule-on-time and wire tension were varied during the experiments. The pule-on-time controls the heat input and the wire tension controls the flexibility of the wire i.e. the mechanical ability to carry on the process steadily [37-39]. The machining speed, kerf width, surface roughness and wire wear were investigated in this study.

\section{Results and discussions}

The influence of pule-on-time on the machining speed is presented in Fig. 1. The figure shows that the machining speed rises with the rise of pule-on-time. The pule-on-time controls the heat generation and spark formation. The increase of pule-on-time (i) increases the heat generation which improves the material removal and (ii) extends the heat for longer time which helps to remove material efficiently. Thus, longer pule-on-time increases the MRR. 
The Fig. 1 also shows that at shorter pule-on-time the influence of wire tension is negligible. However, with the rise of the pule-on-time the trends of the increase of MRR become different. The MRR at lower wire tension is much higher than that of higher wire tension at higher pule-on-time. The increase of MRR is nearly linearly proportional to the rise of puleon-time at higher tension (1800 gf). However, at lower wire tension (600 gf), the rate of rise of MRR is significantly higher with the initial increase of the pule-on-time but it reduces with the further increase of the pule-on-time and becomes almost parallel to the curve of the MRR at higher tension and pule-on-time.

Fig. 2 presents the influence of tension in wire on the MRR at longer and shorter pule-ontimes. It is already known that the lower wire tension and longer pule-on-time increase the MRR. For the ranges of parameters considered in this study, it is seen that at longer pule-ontime, the material removal is notably high at low wire tension. The MRR does not vary much with the initial increase of wire tension. But it decreases significantly with the further rise of tension in wire electrode. On the other hand, at shorter pulse-on-time, the MRR rises with the rise of wire tension but it reduces with the further rise of wire tension.

An ionized waterway among the nearby points of the workpiece and the wire electrode is generated as the voltage is applied at the start of the wire EDM process. Then discharge begins with a high intensity of current which reduces the resistance of the ionized waterway progressively. The heavy flow of current supplements the channel with ions and generates a very strong magnetic arena which compresses the ionized passage and causes local rise of temperature. The temperature in the electrodes increases locally and goes beyond the boiling temperature of the workpiece even with very short spark duration. Thus erosion of the workpiece and wire electrode occurs locally because of the softening and vaporization [40]. The MMR is mainly determined by the quantity of heat input (i.e. temperature and the time length of temperature). Thus, it is very natural that the longer pule-on-time will have higher material removal rate than that of shorter pulse-on-time [41]. The wire tension affects the flexibility and deformation of wire electrode. The wire electrode is more flexible and doesn't deform at lower tension. Thus it will have longer and wider area to take part in material removal. On the other hand, the wire becomes very stiff and the diameter will be reduced at higher tension. This results in shorter and narrower area to take part in material removal which reduces the material removal rate at higher tension. In addition, too much tension in the wire electrode may deteriorate its physical properties and reduce the capacity to produce expected spark. On the other hand, too much flexibility in the wire may not be able to maintain required spark 
gap along its length for efficient material removal. The above discussion clearly shows that the rise of pulse-on-time increases the MRR and the rise of tension in wire reduces the MRR. When both of these input parameters vary simultaneously, the combination of these two parameters to obtain highest MRR is different. For example, the maximum material removal rate occurs at $1200 \mathrm{gf}$ when the pulse-on-time is $2 \mu$ s and the same occurs within 600 to 1200 gf when the pulse-on-time is $4 \mu \mathrm{s}$ in this investigation.

Thermal spalling removes ceramic particles and the surfaces of MMC generated from EDM have the recast layer, surface waviness and blisters because of the plasma collapse at the time of pulse off [33]. The surface of SiCp reinforced Al6061 MMC after machining by WEDM is full of micro-pores, shallow holes, micro-fissures, and drops of debris [42]. Thus distinct difference on the appearance of the machined surfaces for different machining condition is not apparent. However the surface roughness $(\mathrm{Ra})$ varies with the variation of the machining parameters as the dimensions of the craters generated on the machined surface are primarily affected by discharge energy. Fig. 3 demonstrates the influence of pule-on-time on roughness with various wire tension. It demonstrates that initially, the surface roughness $(\mathrm{Ra})$ rises as pule-on-time rise of but it reduces with the further rise of the pule-on-time for higher as well as lower wire tensions. The higher wire tension gives higher roughness at shorter pule-ontime but the lower wire tension gives higher roughness at longer pule-on-time.

The influence of tension in electrode wire on surface roughness at different pule-on-time is presented in Fig. 4. It shows that at lower tension in wire, the roughness (Ra) at pule-on-time $4 \mu \mathrm{s}$ is higher than that of pule-on-time $2 \mu \mathrm{s}$. With the rise of tension in wire, the roughness at $4 \mu$ s starts to decrease with a slight increase at the beginning and the surface roughness at 2 $\mu$ starts to increase for the range of the parameters considered in this investigation. It looks that with the further rise of the tension in wire the roughness at 4 and $2 \mu$ s will intersect and then the surface roughness at $2 \mu$ s will be higher than that of $4 \mu \mathrm{s}$.

The smaller reinforced particles have lower capacity to protect the matrix material from melting during EDM process. Further, the roughness after machining is much higher than the average size of the reinforced particles. Thus, it is likely that the influence of reinforcement particles on the surface finish is negligible and the quality is mainly affected by machining parameters. The surface produced by EDM in Fig. 5 supports the above statements. The similar surfaces were produced under all the machining conditions considered in this study. 
The figure shows that the machined surface is made of micro-pores, micro-fissures, craters, splash of melted material and globules of debris.

At shorter pulse-on-time, lesser heat is generated and the size of the craters becomes smaller and the deposition of the splash material is lower. This causes lower roughness. When the pulse-on-time is longer the surface craters become bigger and amount of the splash material is higher. These cause rougher surface due to longer pulse-on-time. The rise of pule-on-time reduces the plasma flushing efficacy, which sequentially decreases the melted material evicted from the molten pools. This leaves behind the melted material consequently in pools and resolidifies to produce a recast layer. Furthermore, the rise of pulse-on-time also rises the conducted heat to the surface layers below the machined surfaces [33]. However, with the further rise of pulse-on-time causes much bigger craters which merged together, and the deposited the splash material are also denser. Thus surface roughness starts to decrease. The formation of clusters or uneven distribution of reinforced particles deteriorates the properties of the machined surface [43].

It is already mentioned in the last section that the effectiveness of the wire electrode on material removal depends on the wire tension. The same holds true for the roughness of machined surface. The optimum wire tension to achieve minimum surface roughness is influenced by the pulse-on-time. The flexible wire with lower tension covers longer machining length and, smoothly and slowly removes material with better surface finish at shorter pulse-on-time,. With the rise of the tension in wire, the less flexible wire covers shorter machining length and the material is removed by a harder touch at lower energy of the wire electrode. This makes the worse surface finish at higher tension when pulse-on-time is 2 $\mu$ s. At higher pulse-on-time $4 \mu \mathrm{s}$, the wire at low tension may produce unwanted and abnormal sparking as it is difficult to maintain proper spark gape using a flexible wire when higher energy is released. In this case, the stability of the EDM process rises with the rise of the wire tension. The harder touch of the wire at higher tension with higher energy generates smoother surface. A multi-cutting arrangement where first cutting and finish cutting are performed sequentially, can be used to reduce the surface roughness to the fraction of a micron [42].

The kerf width of EDM is the actual width of the slot after performing the machining process. Fig 6 presents the impact of pulse-on-time on the kerf width at lower and higher wire tensions. It shows that the width of the kerf at the top is always bigger than that at the top 
except at very low wire tension and pule-on-time. It is interesting to note that at higher wire tension the kerf widths at top and bottom are in parallel and increase linearly with the increase of pule-on-time. On the other hand, the kerf widths are almost equal at lower wire tension when the pule-on-time is lower. The kerf width at top becomes bigger than that at the bottom due to rise of the pulse-on-time. In this case, the rate of increase of kerf widths is much higher than that at higher wire tension. With the further increase of the pule-on-time the widths of kerf at the top and bottom (Fig. 6) become parallel to each other and continue as horizontal lines.

The influence of tension in wire on the widths of kerf at the top and bottom with the variation of pule-on-time is shown in Fig. 7. It shows that at higher pule-on-time the kerf width at the top is always higher than that at the bottom. Both of these increase with the initial rise of tension in wire but it starts to decrease with further rise of tension in wire. At lower pule-ontime, the top and bottom widths of the kerf are almost equal at low wire tension but with the rise of the tension in wire the top width of kerf starts to increase and the bottom width of the kerf starts to decrease. At a stage the difference between the top and bottom widths becomes maximum. With the further rise of the tension in wire, the top width of the kerf starts to decrease and bottom width of the kerf starts to increase. This reduces the difference between top and bottom widths of the kerf.

The taper shaped slots during EDM process are generated due to wear of the wire electrode. A virgin wire electrode had $250 \mu \mathrm{m}$ diameter at the start of the EDM process. During the machining process the wire starts to wear out and the diameter reduces continuously due to high temperature. Finally, the wire electrode with a reduced diameter comes out from the bottom of the slot. Therefore, the higher wire diameter at the entry of the slot produces larger slot width. On the other hand, the slot width reduces at the bottom of the slot due the smaller wire diameter at the exit. The amount of wear or the diameter reduction of the wire depends on the machining condition. At higher wire tension, the widths of kerf at the top and bottom increase linearly with the rise of pules-on-time because increase of energy. It is exciting to find that the amount of wear in wire didn't increase with the rise of pulse-on-time at higher wire tension. This might be due to the shorter height $(9 \mathrm{~mm})$ of the slot. At lower wire tension, shorter pulse-on-time gives lower energy and produces almost no wear in the wire as the machining process goes slowly and smoothly. The widths of kerf at the top and bottom increase significantly due to the longer pulse-on-time. In this case, the difference between the top and bottom widths increases notably until pulse-on-time $3 \mu$ s. This indicates the rise of 
the wear of the wire electrode. The further rise of the pulse-on-time makes the wear almost constant.

At higher pulse-on-time, the greater difference between top and bottom widths of the slot at lower wire tension indicates higher wire wear compare to that at higher tensions. On the other hand, at shorter pulse-on-time the highest wear in the wire electrode is noted at the medium tension in wire. It is already mentioned and noted that the different combination of wire tension and pulse-on-time affects the EDM process differently, for example shorter pulse-ontime and medium tension in wire gives maximum material removal when pulse-on-time is constant. For the similar reasons, highest wear of the wire (or maximum difference between top and bottom widths of slot) is noted at medium wire tension when the pulse-on-time is constant at $2 \mu \mathrm{s}$.

In this part, the wire diameter refers to the size of the wire just after EDM process which is mainly the wire condition just at the exit. During EDM of MMCs, the variation of the electrode wire diameter after machining is very frequent over a wide range. This might be due to discontinuous sparking during EDM and presence of discontinuous smaller reinforcements in the MMC. To facilitate the understanding of the size variation of wire electrode, the lowest and highest diameters, and picture wire surface obtained in each experiment are presented in Figs. 8 and 9.

Fig. 8 shows the influence of pule-on-time on wire diameter and appearance of surface at lower and higher wire tensions. Though, it is well accepted that the width of the electrode wire decreases due to EDM, in few instance the diameter of the wire increased from $250 \mu \mathrm{m}$ (diameter of the virgin wire electrode) to $280 \mu \mathrm{m}$ during machining of MMCs reinforced with smaller (sub-micron) particles.

The Fig. 8 shows the presence of coatings on the wire when the diameter was increased and this happened at lower wire tension and pulse-on-time. It seems that at lower wire tension and shorter pule-on-time, the wire moves closely to the workpiece material as the kerf width is smaller. This allows the droplets of matrix material to coat the wire during sparking due to spattering of molten metal. In addition, the existence of the higher temperature is for very short time when prevents further melting of the deposited matrix material. At the same wire tension when the pule-on-time increases a mix of coated and uncoated wire was noted. In this case, the coated wire was of higher diameter and uncoated wire of lower diameter. The maximum variation of wire diameter was noted at this instance. With the further increase of 
the pule-on-time, all the wire samples were free of coating and the variation of wire diameter was minor. All the wire surfaces were free of coating at higher wire tension at all the puleon-time considered in this study. The diameter variation was almost constant at higher wire tension with the rise of pule-on-time.

Fig. 9 demonstrates the variation of the wire diameter at higher and lower pule-on-time with the variation of wire tensions. This figure clearly shows that the difference between maximum and minimum diameters at low tension and short pule-on-time is minor as all the surfaces are coated with the matrix material. The major difference between the maximum and minimum diameters was at medium wire tension at 1200 gf. Irrespective of pule-on-time, sometimes the coating appears and sometimes it does not, at this tension. Therefore, the wide variations of the wire diameter are noted. The variation of the wire diameter was reduced with the further increase of the wear tension, and at this stage no coating is noted on the wire electrode.

The wire tension affects the flexibility of the wire electrode. At lower tension, the wire is more flexible and it is easy for any material to attach on it. Thus, the matrix material coats the wire surface at low pule-on-time. However, at longer pule-on-time, the generated heat stays longer and removes the coated material. Thus, coating disappears at higher pule-on-time even at low wire tension. It is surprising to see the presence of coating at higher tension (1200 gf) and higher pule-on-time (4 $\mu \mathrm{s})$ (Fig. 9). The EDM is a discrete processes and MMC is nonhomogeneous material. Thus, the material removal process is not always uniform. This unusual results may be because of uncontrolled spark, and variation of electrolyte concentration and flush rate due to clogging by reinforced particles [27].

\section{Conclusion}

The in-depth investigation of the wire EDM of 6061 aluminium alloy reinforced with $700 \mathrm{~nm}$ $\mathrm{SiC}$ particles provides the following conclusions:

(a) The longer pule-on-time generates more heat and facilitates material removal rate. Similar to any other materials the rise of pule-on-time increases the MRR for smaller particle reinforced 6061 aluminium alloy MMC. The contribution of tension in wire on the MRR is negligible at shorter pule-on-time but higher material removal rate is noted at higher pule-on-time. 
(b) The pule-on-time and wire tension affects the surface roughness in a complex way. Generally the shorter pule-on-time gives better surface finish at lower wire tension but longer pule-on-time gives better surface finish at higher wire tension. There is trend that a similar surface roughness will be achieved higher wire tension.

(c) Width of the kerf at bottom is always smaller than that of top. Both the top and bottom widths of kerfs increase with the rise of pule-on-time. However, the trend of increase is different at different wire tension. The difference between the top and bottom widths of the slot shows the wear of wire electrode.

(d) A significant variation of the wire diameter was caused during machining of $700 \mathrm{~nm} \mathrm{SiC}$ particles reinforced 6061 aluminium alloy. The variation was mainly caused by the presence or absence of the matrix material coating on the wire. The coating of matrix material is generally noted at lower wire tension and shorter pule-on-time. The variation of the wire diameter may also be introduced by clogging of small reinforced particles which causes uncontrolled spark and variation of electrolyte’s ability.

(e) Further investigation is required to predict the surface finish and over cutting of MMCs after wire EDM process.

\section{References}

1. Pramanik, A., et al., Ultraprecision turning of electroless nickel: effects of crystal orientation and origin of diamond tools. The International Journal of Advanced Manufacturing Technology, 2009. 43(7-8): p. 681-689.

2. Pramanik, A., Islam, M. N., Basak, A., \& Littlefair, G. , Machining and Tool Wear Mechanisms during Machining Titanium Alloys. Advanced Materials Research, 2013. 651: p. 338-343.

3. Pramanik, A., L. Zhang, and J. Arsecularatne, Micro-indentation of metal matrix composites —an FEM analysis. Key Eng Mater, 2007. 340: p. 341.

4. Pramanik, A., L.C. Zhang, and J.A. Arsecularatne, Deformation mechanisms of MMCs under indentation. Composites Science and Technology, 2008. 68(6): p. 13041312.

5. Ho, K.H. and S.T. Newman, State of the art electrical discharge machining (EDM). International Journal of Machine Tools and Manufacture, 2003. 43(13): p. 1287-1300.

6. Abrate, S., Walton, D., Machining of composite materials. Part II: Non-traditional methods. Composites Manufacturing, 1992. 3(2): p. 85-94.

7. Ramesh, S., N. Natarajan, and V. Krishnaraj, Experimental investigation of Al6061/SiCp/B4Cp hybrid MMCs in wire electrical discharge machine. INDIAN JOURNAL OF ENGINEERING AND MATERIALS SCIENCES, 2014. 21(4): p. 409-417.

8. Pramanik, A., Developments in the non-traditional machining of particle reinforced metal matrix composites. International Journal of Machine Tools and Manufacture, 2014. 86(0): p. 44-61. 
9. Singh, B., J. Kumar, and S. Kumar, Influences of Process Parameters on MRR Improvement in Simple and Powder Mixed EDM of AA6061/10\% SiC Composite. Materials and Manufacturing Processes, 2014(just-accepted).

10. Sidhu, S.S., A. Batish, and S. Kumar, Study of surface properties in particulatereinforced metal matrix composites (MMCs) using powder-mixed electrical discharge machining (EDM). Materials and Manufacturing Processes, 2014. 29(1): p. 46-52.

11. Ramasawmy, H. and L. Blunt, Effect of EDM process parameters on 3D surface topography. Journal of Materials Processing Technology, 2004. 148(2): p. 155-164.

12. Rao, T.B. and A.G. Krishna, Principal Component Analysis of Correlated WEDM Performance Characteristics during Machining ZC63/SiCP MMCs. Journal for Manufacturing Science and Production, 2014. 14(1): p. 13-21.

13. Liu, J.W., T.M. Yue, and Z.N. Guo, Wire Electrochemical Discharge Machining of Al2O3 Particle Reinforced Aluminum Alloy 6061. Materials and Manufacturing Processes, 2009. 24(4): p. 446-453.

14. Meena, K., A. Manna, and S. Banwait, Effect of wire feed rate and wire tension during machining of PR-AL-SiC-MMC's by WEDM. European Journal of Engineering and Technology, 2013. 1(1).

15. Maher, I., A.A. Sarhan, and M. Hamdi, Review of improvements in wire electrode properties for longer working time and utilization in wire EDM machining. The International Journal of Advanced Manufacturing Technology, 2014: p. 1-23.

16. Saini, V., A.K. Zahid, and A.N. Siddiquee, Optimization of wire electric discharge machining of composite material (Al6061/Sicp) using taguchi method. International Journal of Mechanical and Production Engineering, 2013. 2.

17. Pramanik, A., L.C. Zhang, and J.A. Arsecularatne, Prediction of cutting forces in machining of metal matrix composites. International Journal of Machine Tools and Manufacture, 2006. 46(14): p. 1795-1803.

18. Liu, J.W. and Y.Z. Wu, An Orthogonal Experimental Analysis of WEDM-HS of Al2O3 Particle-Reinforced Aluminum Alloy 6061 with 10-Vol\% Al2O3. Advanced Materials Research, 2014. 910: p. 61-64.

19. Lal, S., et al., An investigation on effects of wire electrical discharge machining parameters on surface roughness of newly developed hybrid metal matrix composite. Proceedings of the Institution of Mechanical Engineers, Part B: Journal of Engineering Manufacture, 2013: p. 0954405413506703.

20. Muller, F., J. Monaghan, and I. Verein Deut, Electro discharge machining of a particle reinforced metal matrix composite, in 12th International Symposium for Electromachining. 1998. p. 513-522.

21. Seo, Y., D. Kim, and M. Ramulu, Electrical discharge machining of functionally graded 15-35 vol\% SiCp/Al composites. Materials and Manufacturing Processes, 2006. 21(5): p. 479-487.

22. Hung, N.P., L.J. Yang, and K.W. Leong, Electrical discharge machining of cast metal matrix composites. Journal of Materials Processing Technology, 1994. 44(3-4): p. 229-236.

23. Gatto, A. and L. Iuliano, Cutting mechanisms and surface features of WED machined metal matrix composites. Journal of Materials Processing Technology, 1997. 65(1-3): p. 209-214.

24. Rozenek, M., et al., Electrical discharge machining characteristics of metal matrix composites. Journal of Materials Processing Technology, 2001. 109(3): p. 367-370.

25. Yan, B.H., et al., Examination of wire electrical discharge machining of $\mathrm{Al}(2) \mathrm{O}(3) \mathrm{p} / 6061 \mathrm{Al}$ composites. International Journal of Machine Tools \& Manufacture, 2005. 45(3): p. 251-259. 
26. Patel, K., P.M. Pandey, and P.V. Rao, Understanding the role of weight percentage and size of silicon carbide particulate reinforcement on electro-discharge machining of aluminium-based composites. Materials and Manufacturing Processes, 2008. 23(7): p. 665-673.

27. Saha, P., Tarafdar, D., Pal, S. K., Saha, P., Srivastava, A. K., \& Das, K. , Modeling of wire electro-discharge machining of TiC/Fe in situ metal matrix composite using normalized RBFN with enhanced k-means clustering technique. The International Journal of Advanced Manufacturing Technology, 2009. 43(1-2): p. 107-116.

28. Agrawal, S.S. and V. Yadava, Modeling and prediction of material removal rate and surface roughness in surface-electrical discharge diamond grinding process of metal matrix composites. Materials and Manufacturing Processes, 2013. 28(4): p. 381-389.

29. Guo, Z.N., et al., Experimental investigation into shaping particle-reinforced material by WEDM-HS. Journal of Materials Processing Technology, 2002. 129(1-3): p. 56-59.

30. Patil, N.G., Brahmankar, P. K. , Some investigations into wire electro-discharge machining performance of Al/SiC $p$ composites. International Journal of Machining and Machinability of Materials, 2006. 1(4): p. 412-431.

31. Manna, A. and B. Bhattacharyya, Taguchi and Gauss elimination method: A dual response approach for parametric optimization of CNC wire cut EDM of PRAISiCMMC. International Journal of Advanced Manufacturing Technology, 2006. 28(1-2): p. 67-75.

32. Sivaprakasam, P., P. Hariharan, and S. Gowri, Optimization of Micro-WEDM Process of Aluminum Matrix Composite (A413-B4C): A Response Surface Approach. Materials and Manufacturing Processes, 2013. 28(12): p. 1340-1347.

33. Kumar, S.S., et al., Electrical Discharge Machining of Al (6351)-SiC-B4C Hybrid Composite. Materials and Manufacturing Processes, 2014. 29(11-12): p. 1395-1400.

34. Yue, T., Y. Dai, and W. Lau, An examination of wire electrical discharge machining (WEDM) of Al2O3 particulate reinforced aluminum based composites. MATERIAL AND MANUFACTURING PROCESS, 1996. 11(3): p. 341-350.

35. Basak, A., A. Pramanik, and M. Islam, Failure Mechanisms of Nanoparticle Reinforced Metal Matrix Composite. Advanced Materials Research, 2013. 774: p. 548-551.

36. Pramanik, A. and G. Littlefair, Fabrication of Nano-Particle Reinforced Metal Matrix Composites. Advanced Materials Research, 2013. 651: p. 289-294.

37. Hwa Yan, B., et al., Examination of wire electrical discharge machining of $\mathrm{Al}_{2} \mathrm{O}_{3}$ p/6061Al composites. International Journal of Machine Tools and Manufacture, 2005. 45(3): p. 251-259.

38. Liao, Y.S., J.T. Huang, and H.C. Su, A study on the machining-parameters optimization of wire electrical discharge machining. Journal of Materials Processing Technology, 1997. 71(3): p. 487-493.

39. Spedding, T.A. and Z.Q. Wang, Study on modeling of wire EDM process. Journal of Materials Processing Technology, 1997. 69(1-3): p. 18-28.

40. Scott, D.A.N., S. Boyina, and K.P. Rajurkar, Analysis and optimization of parameter combinations in wire electrical discharge machining. International Journal of Production Research, 1991. 29(11): p. 2189-2207.

41. Hasçalık, A. and U. Çaydaş, Electrical discharge machining of titanium alloy (Ti6Al-4V). Applied Surface Science, 2007. 253(22): p. 9007-9016.

42. Zhenlong, W., et al., Surface Integrity Associated with SiC/Al Particulate Composite by Micro-Wire Electrical Discharge Machining. Materials and Manufacturing Processes, 2014. 29(5): p. 532-539. 
43. Sidhu, S.S., A. Batish, and S. Kumar, EDM of metal matrix composite for parameter design using lexicographic goal programming. Materials and Manufacturing Processes, 2013. 28(4): p. 495-500.

\section{Figures and tables}

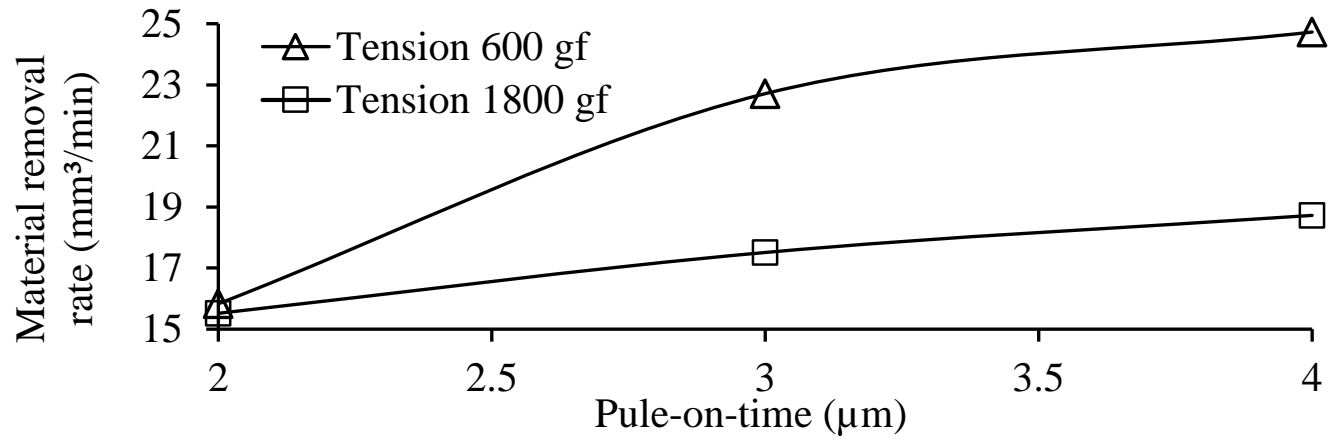

Fig. 1 Influence of pule-on-time on the MRR at high as well as low wire tensions

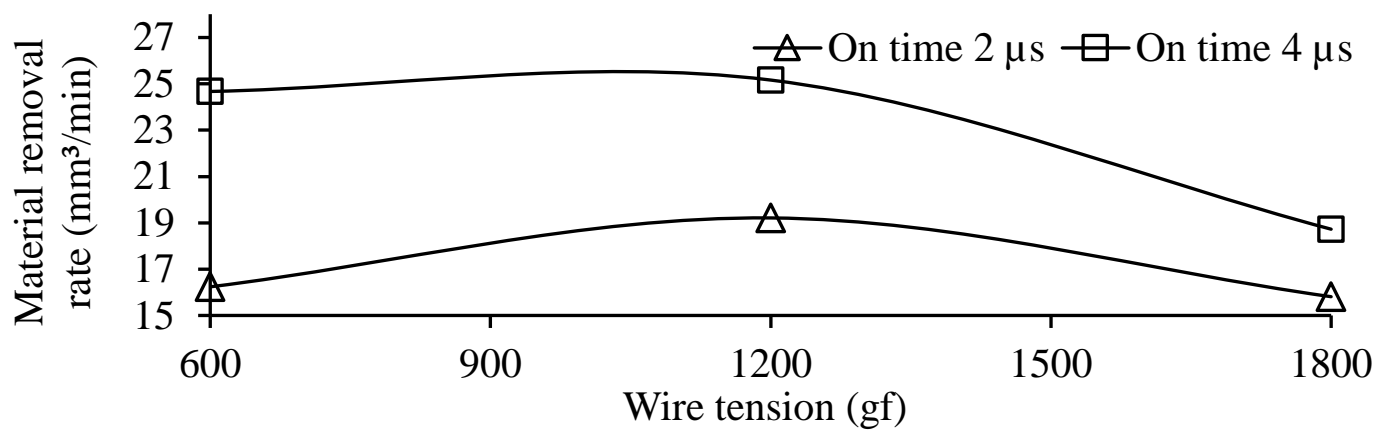

Fig. 2 Variation of material removal rate with wire tension at longer and shorter pule-ontimes

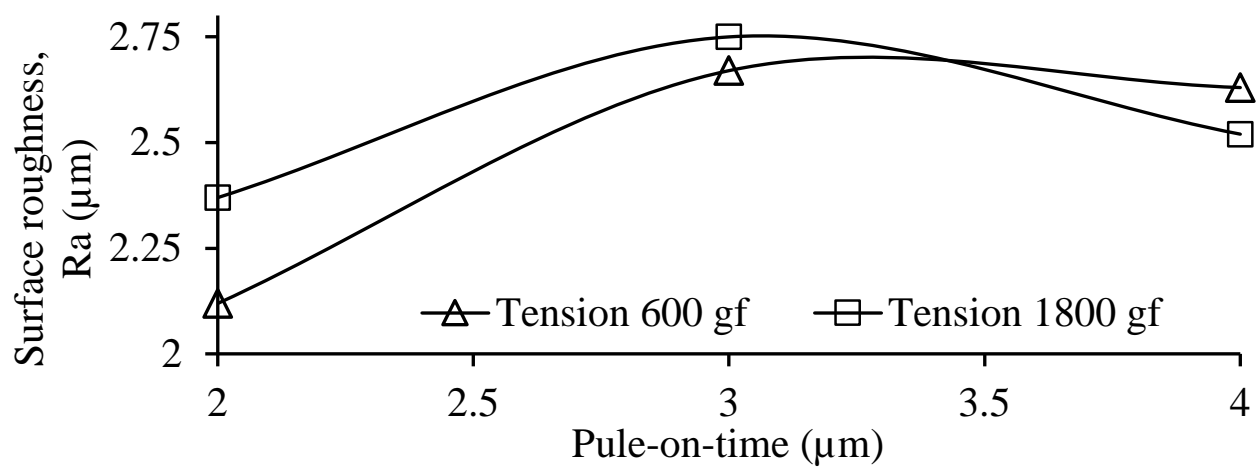

Fig. 3 Variation of surface roughness with pule-on-time at higher and lower wire tensions 


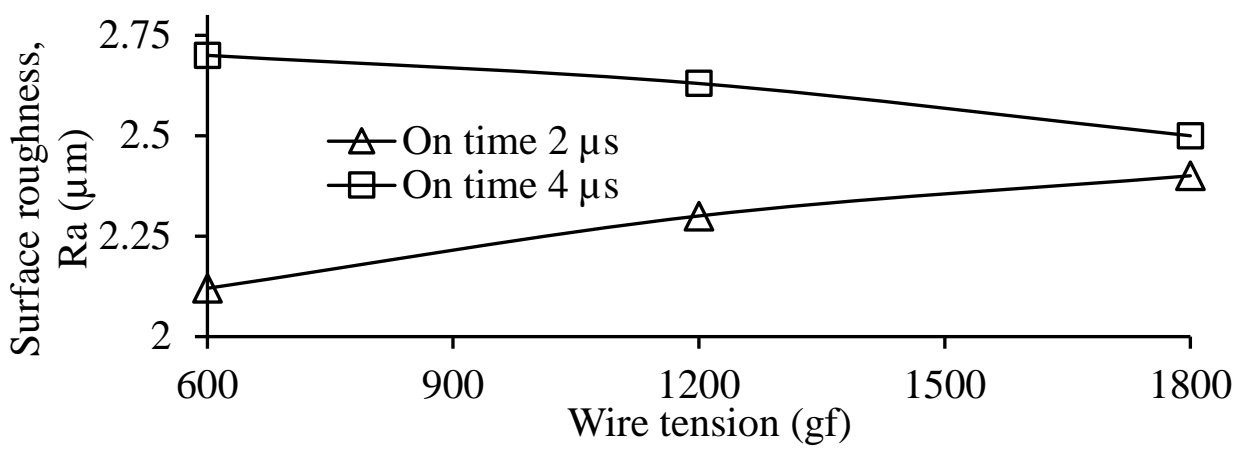

Fig. 4 Variation of surface roughness with wire tension at high and low pule-on-times

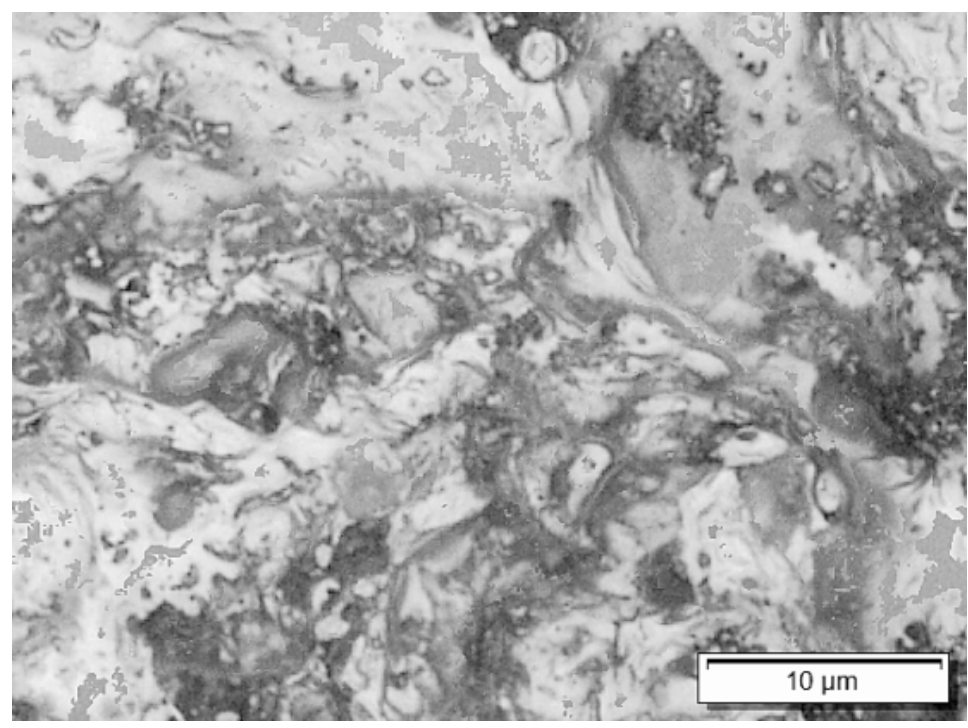

Fig. 5 Surface of $700 \mathrm{~nm} \mathrm{SiC} \mathrm{reinforced} 6061$ aluminium alloy produced by EDM (from experiment no. 9).

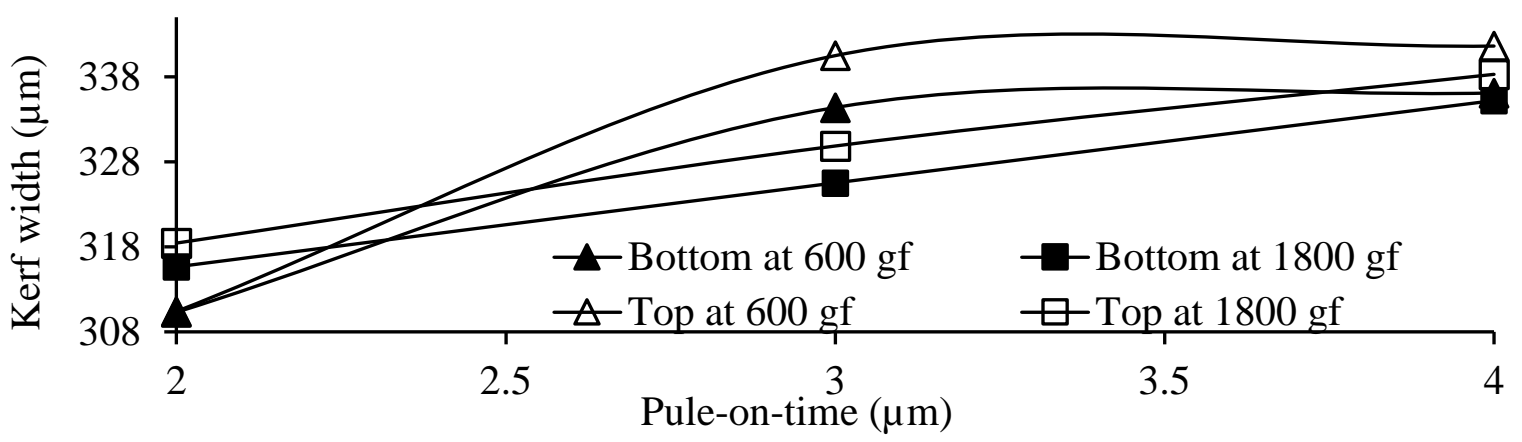

Fig. 6 Influence of pulse-on-time on kerf width at high and low wire tensions 


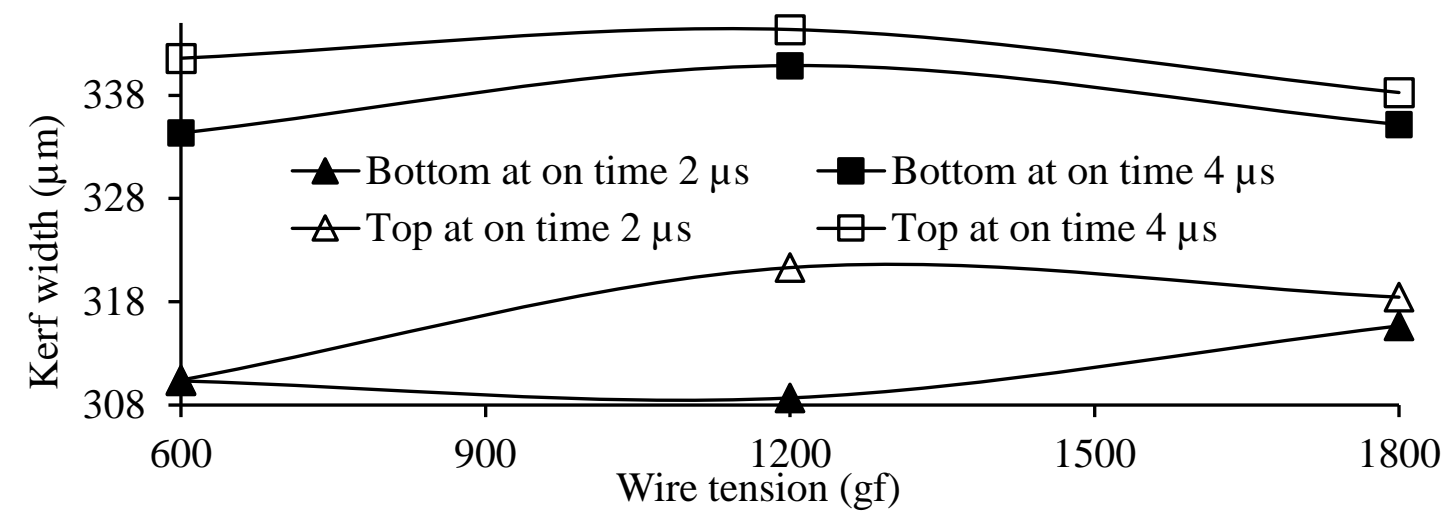

Fig. 7 Effect of wire tension on kerf width at high and low pule-on-times

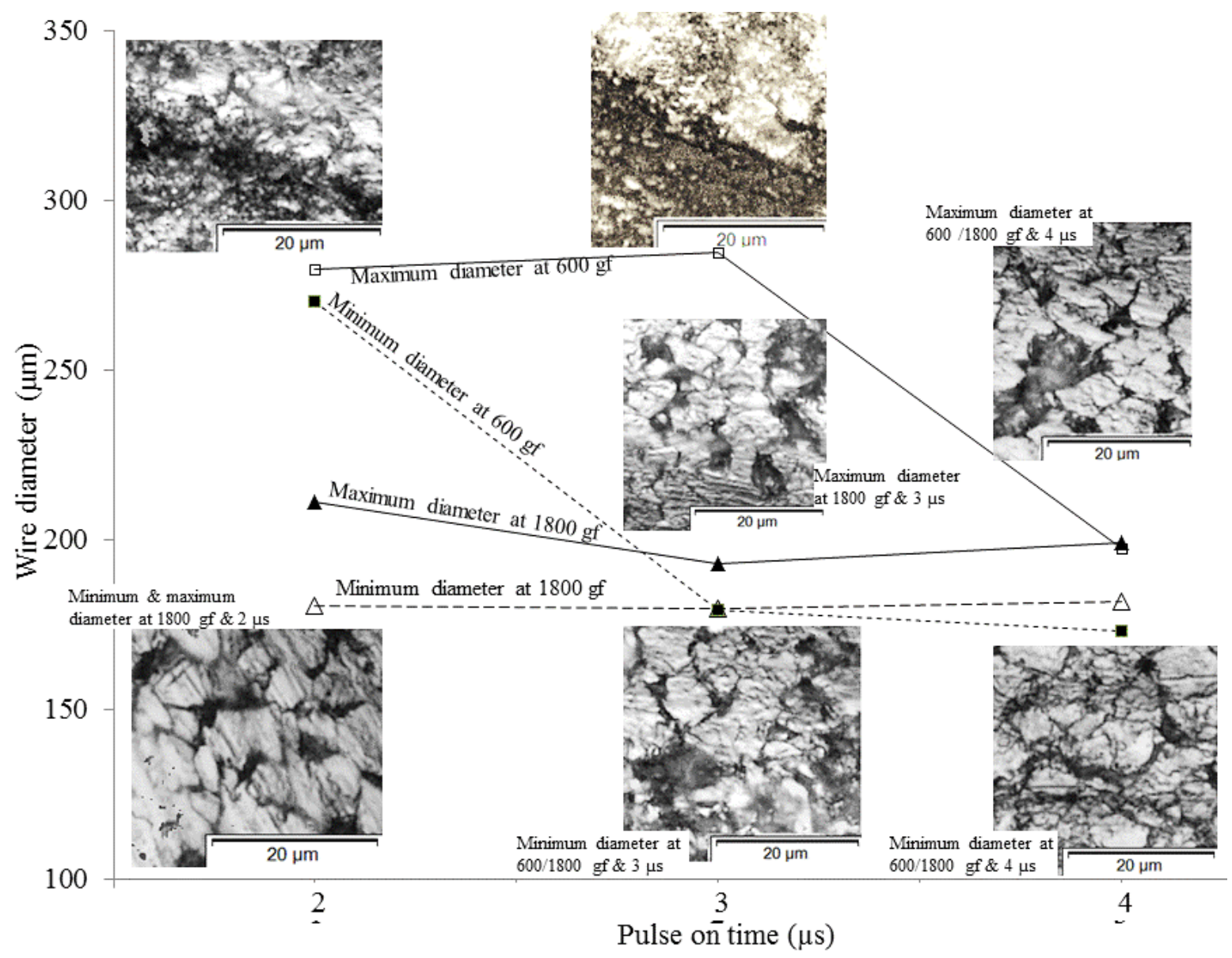

Fig. 8 Influence of pule-on-time on wire diameter 


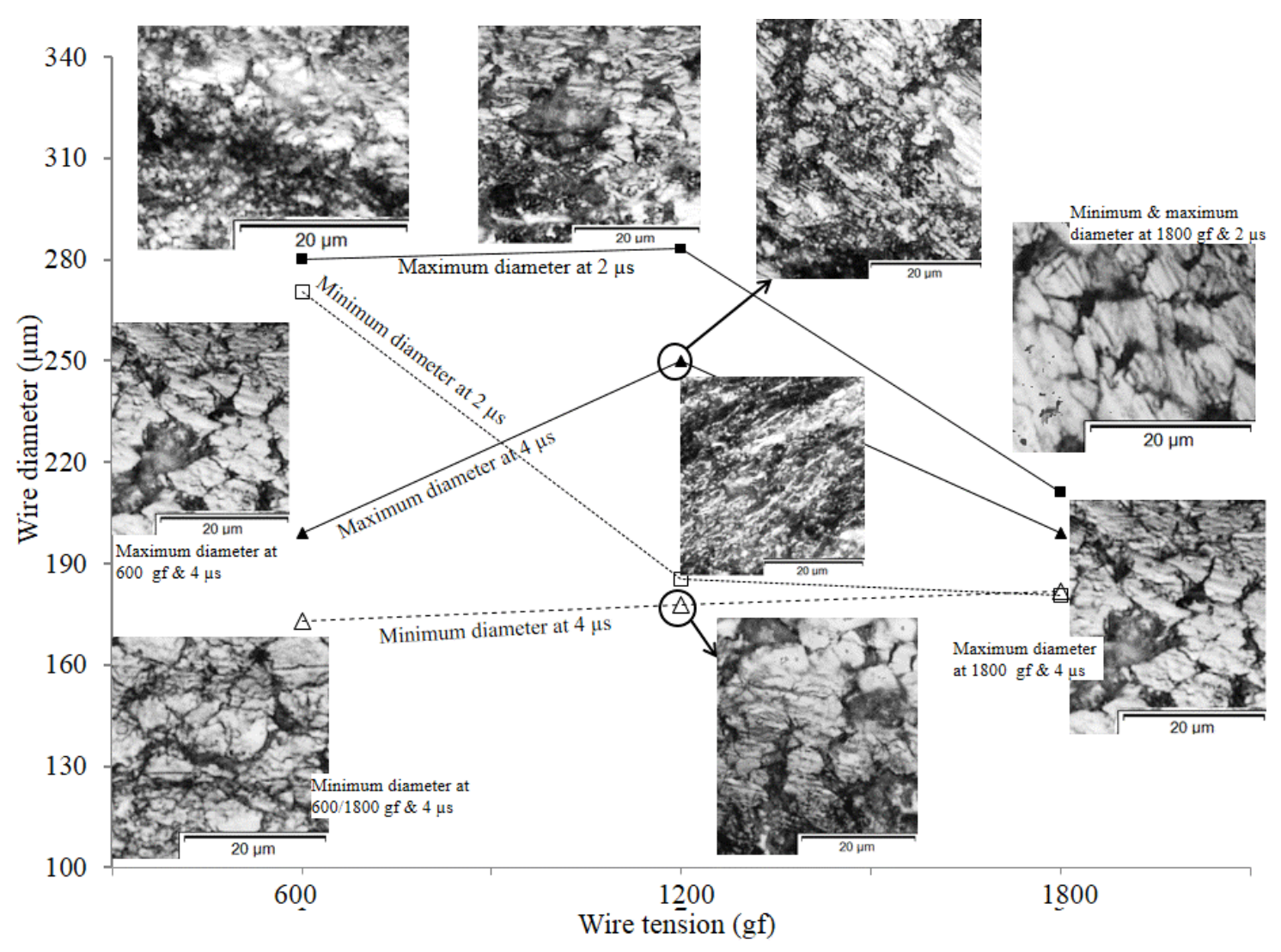

Fig. 9 Influence of wire tension on the wire diameter

Table 1 Experimental conditions

\begin{tabular}{|c|c|c|}
\hline Experiment no. & Wire tension (gf) & Pule-on-time $(\mu \mathrm{s})$ \\
\hline 1 & 600 & 2 \\
\hline 2 & 600 & 3 \\
\hline 3 & 600 & 4 \\
\hline 4 & 1800 & 2 \\
\hline 5 & 1800 & 3 \\
\hline 6 & 1800 & 4 \\
\hline 7 & 1200 & 2 \\
\hline 8 & 1200 & 4 \\
\hline 9 & 1800 & 4 \\
\hline
\end{tabular}

